\title{
On the Convergence of the Iterative Gauss-Seidel-Based Electric Field Algorithm for the Solution of Antenna Array Mutual Coupling
}

\author{
Tomislav Marinović ${ }^{1,2 *}$, Mario Kuprešak ${ }^{1}$, Rob Maaskant ${ }^{2,3}$, and Guy A. E. Vandenbosch ${ }^{1}$ \\ ${ }^{1}$ Dept. of Electrical Engineering (ESAT), KU Leuven, 3001 Leuven, Belgium \\ ${ }^{2}$ Dept. of Electrical Engineering, Chalmers University of Technology, 41296 Gothenburg, Sweden \\ ${ }^{3}$ Dept. of Electrical Engineering, Eindhoven University of Technology, 5612 AZ Eindhoven, The Netherlands \\ * Corresponding author, email: tomislav.marinovic@esat.kuleuven.be
}

\begin{abstract}
Recently, a new method for the solution of the antenna array mutual coupling (MC), which is based on the field data of an isolated element and relates to the iterative Gauss-Seidel method, has been proposed. In this paper, we study the convergence of the proposed method by comparing it with the convergence of the classical Gauss-Seidel iteration applied to a method-of-moments (MoM) system. Despite several approximations being used in the field-based method, similar behavior between the two methods has been observed, both in terms of the stability and the rate of the convergence.
\end{abstract}

Keywords-Antenna arrays, mutual coupling (MC), domaindecomposition methods (DDMs), iterative methods.

\section{INTRODUCTION}

Finite antenna arrays are used in various communication and sensing systems. An important family of the solution methods for the MC in finite antenna arrays involves the DDMs in which the effect of MC is determined by modeling the interactions between the smaller partitions of the entire array geometry which, computationally speaking, can be solved relatively inexpensively. Recently, a novel domaindecomposition-based solution strategy for finite antenna arrays has been proposed [1], [2], which employs the sampled far-field data of an isolated element and may be modeled based on the iterative Gauss-Seidel method. Here, mutual interactions between the array elements are modeled by approximating the incident spherical wave expansion (SWE)based field scattered from other array elements as a single plane wave with mutually-orthogonal polarization. In the initial numerical convergence analysis performed in [2], the dependency of the convergence on the element size, array size and spacing has been studied. However, due to several approximations which are used in this method (the use of the far-field data, the SWE modal truncation and the single-plane-wave approximation a.o.), the convergence of this method might not be entirely consistent with the convergence of the classical Gauss-Seidel method applied to a MoM matrix system, which can be analyzed theoretically. We address this research question in the following study.

\section{TheORETICAL DEVElopMENT}

The mutually-coupled electric far field radiated by element $m$ in iteration $q \geq 1$ can be expressed using the iterative Gauss-Seidel-based method as [2]

$$
\underline{\boldsymbol{F}}_{m}^{(q)}=\underline{\boldsymbol{F}}_{m}^{(0)}+\sum_{m^{\prime}=1, m^{\prime} \neq m}^{N} \underline{\underline{F}}_{m m^{\prime}}^{\mathrm{s}(0)} \underline{\check{\boldsymbol{w}}}_{m m^{\prime}}^{\left(q_{\mathrm{s}}\right)},
$$

where $\left.\left(q_{\mathrm{s}}=q\right)\right|_{m^{\prime}<m}$ and $\left.\left(q_{\mathrm{s}}=q-1\right)\right|_{m^{\prime}>m}$. Here, $\underline{\boldsymbol{F}}_{m}^{(0)}$ contains the $(\hat{\boldsymbol{\theta}}, \hat{\boldsymbol{\phi}})$-components of the far field of the isolated element $m$ due to a unit-amplitude excitation at its antenna terminals, $\check{\check{\boldsymbol{w}}}_{m m^{\prime}}^{\left(q_{\mathrm{s}}\right)} \in \mathbb{C}^{2 \times 1}$ contains the $(\hat{\boldsymbol{\theta}}, \hat{\boldsymbol{\phi}})$-components of the incident SWE-based field originating from element $m^{\prime}$, evaluated at the reference point of element $m$, while $\underline{\underline{\boldsymbol{F}}}_{m m^{\prime}}^{\mathrm{s}(0)} \in$ $\mathbb{C}^{2 \times 2}$ is the scattering dyad which transforms $\underline{\check{\boldsymbol{w}}}_{m m^{\prime}}^{\left(q_{\mathrm{s}}\right)}$ onto the field scattered by element $m$, as detailed in [2].

Similarly, in the classical MoM matrix system $\boldsymbol{Z} \boldsymbol{J}=\boldsymbol{V}$, the solution for the surface current of element $m$ in iteration $q \geq 1$ can be obtained using the block-based iterative GaussSeidel method as

$$
\boldsymbol{J}_{m}^{(q)}=\boldsymbol{J}_{m}^{(0)}-\sum_{m^{\prime}=1, m^{\prime} \neq m}^{N} \boldsymbol{Z}_{m m}^{-1} \boldsymbol{Z}_{m m^{\prime}} \boldsymbol{J}_{m^{\prime}}^{\left(q_{\mathrm{s}}\right)},
$$

where $\boldsymbol{Z}=\boldsymbol{Z}_{\mathrm{on}}+\boldsymbol{Z}_{\text {off }}$ and $\boldsymbol{V}$ are the impedance matrix and the voltage vector, respectively. Here, $\boldsymbol{Z}_{\text {on }}$ and $\boldsymbol{Z}_{\text {off }}$ contain the intra-element $\left(m=m^{\prime}\right)$ and the inter-element coupling blocks $\left(m \neq m^{\prime}\right)$, respectively. Moreover, $\boldsymbol{Z}_{\text {off }}=$ $\boldsymbol{Z}_{\text {off, } \mathrm{L}}+\boldsymbol{Z}_{\text {off,U, where "L" and "U" denote the lower and upper }}$ triangular parts of the matrix $\boldsymbol{Z}_{\text {off }}$, respectively. A necessary and sufficient condition for the convergence of the iterative Gauss-Seidel method involves the spectral radius $\rho\{\cdot\}$ of the Gauss-Seidel iteration matrix $\boldsymbol{G}=-\left(\boldsymbol{Z}_{\text {on }}+\boldsymbol{Z}_{\text {off,L }}\right)^{-1} \boldsymbol{Z}_{\text {off, } \mathrm{U}}$ $(\rho(\boldsymbol{G})<1)$. In addition, convergence is related to the condition number of the impedance matrix $\boldsymbol{Z}$ which may be defined using the infinity-norm $\|\cdot\|_{\infty}$ as $\kappa_{\infty}(\boldsymbol{Z})=$ $\|\boldsymbol{Z}\|_{\infty} \cdot\left\|\boldsymbol{Z}^{-1}\right\|_{\infty}$. 


\section{NUMERICAL RESUlTS}

To analyze the convergence of the algorithms based on the Gauss-Seidel method, we consider the regular arrays of parallel center-fed half-wavelength strip dipoles while varying the inter-element spacing and the array size. The elements are uniformly-excited by a voltage source of $1 \mathrm{~V}$ at $f_{0}=28 \mathrm{GHz}$. The input data required for Eqs. (1) [2, p. 5] and (2) are obtained using the MoM-based "FEKO" solver with the "triangle edge length" parameter specified as $\lambda_{0} / 60$. The field-based algorithm is implemented according to Eq. (4) of [2], with the convergence residual defined as

$$
\rho_{\mathrm{F}}^{(q)}=\left\|\check{\boldsymbol{W}}^{(q)}-\check{\boldsymbol{W}}^{(q-1)}\right\|_{2} /\left\|\check{\boldsymbol{W}}^{(q)}\right\|_{2},
$$

where $\check{\boldsymbol{W}}^{(q)} \in \mathbb{C}^{2 N^{2}}$ is the weight vector containing the values of $\hat{\boldsymbol{\theta}}$ and $\hat{\boldsymbol{\phi}}$ components of $\underline{\check{\boldsymbol{w}}}_{m m^{\prime}}$ for all $m, m^{\prime}$ in iteration $q$, while $\|\cdot\|_{2}$ represents the $L_{2}$ norm [2]. Similarly, the convergence of (2) is analyzed via

$$
\rho_{\mathrm{J}}^{(q)}=\left\|\boldsymbol{J}^{(q)}-\boldsymbol{J}^{(q-1)}\right\|_{2} /\left\|\boldsymbol{J}^{(q)}\right\|_{2} .
$$

Fig. 1 shows the convergence parameters of the iterative current-based and field-based Gauss-Seidel methods used to analyze the dipole arrays of different size and spacing. Although the method based on the iterative Gauss-Seidel interpretation of the electric fields involves several approximations, as noted in the Introduction, a good agreement between the convergences of these methods can be observed. Only in the case of the $9 \times 9$ array with $0.75 \lambda_{0}$ spacing, the required number of iterations seems to significantly differ in the favour of the current-based method. Possibly, the truncated information content due to the approximations used in the field-based method - the effect more pronounced at this array size and spacing, might be the cause of a slower convergence. Additionally, the inverse relation between the magnitudes of the condition number and the spectral radius, and the required number of iterations to obtain the specified accuracy, can be observed in the results of both methods. Moreover, both methods show the non-convergent behavior for the smallest considered spacing $\left(d=\lambda_{0} / 2\right)$, where the spectral radii are larger than unity. Finally, the convergence rates of both methods for different array sizes and spacing are plotted in Fig. 2, showing comparable progression trends observable in the convergence plots of both methods.

\section{CONCLUSION}

The convergence of the electric-field algorithm based on the Gauss-Seidel method has been analyzed through a comparison with the classical Gauss-Seidel method applied to a MoM matrix system. Despite several approximations being made in the field-based method, similarities in terms of the convergence behavior of the two methods considering the dipole arrays with different sizes and spacing, have been observed. In the future work, we will analyze the impact of the element selection, element size, array excitation and irregular array geometry on the convergence of both methods.
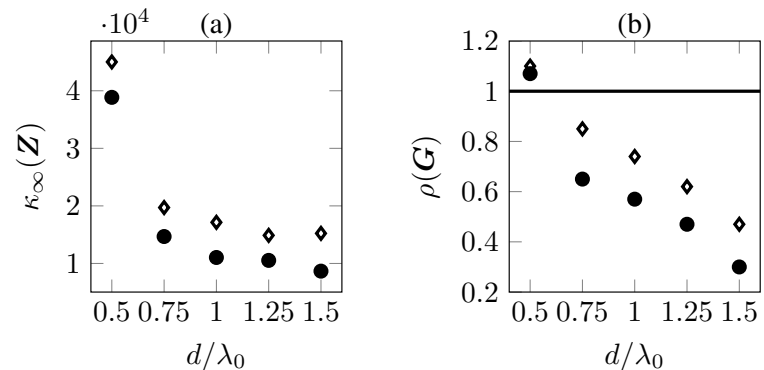

(c)
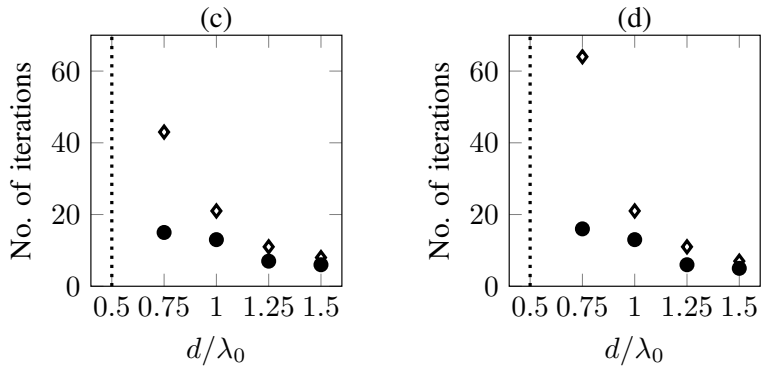

Fig. 1. Convergence parameters of the $5 \times 5$ (circle) and $9 \times 9$ (diamond) arrays of parallel $z$-oriented half-wavelength strip dipoles: (a) condition number $\kappa_{\infty}(\boldsymbol{Z})$; (b) spectral radius $\rho(\boldsymbol{G})$; (c) convergence of the current-based Gauss-Seidel method (2), and (d) convergence of the fieldbased Gauss-Seidel method [2, Eq. 4]. The stop criteria are specified as $\rho_{\mathrm{J}}^{(q)} \leq 0.001$ (c) and $\rho_{\mathrm{F}}^{(q)} \leq 0.001$ (d). The dotted line indicates the non-convergent behavior for both considered array sizes.

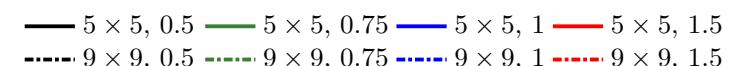

(a)
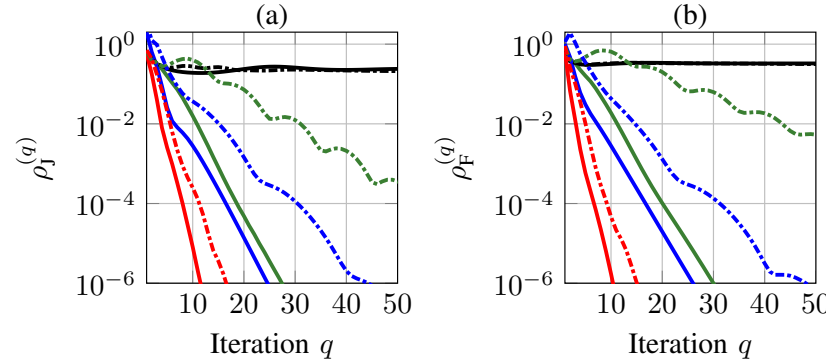

Fig. 2. Relative residuals of the current-based (a) and field-based (b) GaussSeidel iterations considering various arrays of parallel half-wavelength dipoles. The legend lists the array size and spacing in terms of wavelength.

\section{ACKNOWLEDGMENT}

This project has received funding from the European Union's Horizon 2020 research and innovation program under the Marie Skłodowska-Curie grant agreement No. 721732 .

\section{REFERENCES}

[1] D. I. L. de Villiers and R. Maaskant, "Element pattern prediction in mutually-coupled arrays through isolated antenna characterization," in 2017 Int. Symp. Antennas Propag. (ISAP), Oct. 2017, pp. 1-2.

[2] T. Marinovic, D. I. L. de Villiers, D. Bekers, M. N. Johansson, A. Stjernman, R. Maaskant, and G. A. E. Vandenbosch, "Fast characterization of mutually-coupled array antennas using isolated antenna far-field data," IEEE Trans. Antennas Propag., 2020, in press. 